\title{
Non-Lorentzian Local Density of States in Coupled Photonic Crystal Cavities Probed by Near- and Far-Field Emission
}

\author{
Daniele Pellegrino, ${ }^{1}$ Dario Balestri, ${ }^{2,3}$ Nicoletta Granchi, ${ }^{2,3}$ Matteo Ciardi $\odot,{ }^{2}$ Francesca Intonti, ${ }^{2,3}$ \\ Francesco Pagliano, ${ }^{1,4}$ Andrei Yu. Silov, ${ }^{1}$ Frank W. Otten, ${ }^{1}$ Tong Wu, ${ }^{5}$ Kevin Vynck, ${ }^{5}$ \\ Philippe Lalanne $\odot,{ }^{5}$ Andrea Fiore, ${ }^{1}$ and Massimo Gurioli $\oplus^{2,3}$ \\ ${ }^{1}$ Department of Applied Physics and Institute for Photonic Integration, Eindhoven University of Technology, \\ 5600 MB Eindhoven, Netherlands \\ ${ }^{2}$ Department of Physics, University of Florence, via Sansone 1, I-50019 Sesto Fiorentino (FI), Italy \\ ${ }^{3}$ European Laboratory for Nonlinear Spectroscopy, via Nello Carrara 1, I-50019, Sesto Fiorentino (FI), Italy \\ ${ }^{4}$ nanoPHAB, Groene Loper 19, P.O.Box 513, 5612 AP Eindhoven, The Netherlands \\ ${ }^{5}$ LP2N, Institut d'Optique Graduate School, CNRS, Univ. Bordeaux, 33400 Talence, France
}

(Received 16 August 2019; accepted 3 March 2020; published 26 March 2020)

\begin{abstract}
Recent theories proposed a deep revision of the well-known expression for the Purcell factor, with counterintuitive effects, such as complex modal volumes and non-Lorentzian local density of states. We experimentally demonstrate these predictions in tailored coupled cavities on photonic crystal slabs with relatively low optical losses. Near-field hyperspectral imaging of quantum dot photoluminescence is proved to be a direct tool for measuring the line shape of the local density of states. The experimental results clearly evidence non-Lorentzian character, in perfect agreement with numerical and theoretical predictions. Spatial maps with deep subwavelength resolution of the real and imaginary parts of the complex mode volumes are presented. The generality of these results is confirmed by an additional set of far-field and time-resolved experiments in cavities with larger modal volume and higher quality factors.
\end{abstract}

DOI: 10.1103/PhysRevLett.124.123902

The last decades placed nanophotonics at the heart of many fields of research, including integrated photonics [1], optical [2] and quantum sensing [3], metamaterials [4], quantum electrodynamics [5], and topological photonics [6]. The possibility to tailor the line shape and spatial extent of the photonic local density of states (LDOS) $\rho(\mathbf{r}, \omega)$ is one of the major breakthroughs of the past decades $[1,7-$ 9]. The so-called Purcell effect, i.e., the modification of the spontaneous emission (SE) rate, is driven by the quality factor $(Q)$ and the modal volume $(V)$ of the microresonators [10]. The cavity $Q$ factor is nowadays well comprehended; different strategies have been proposed and developed to increase it $[11,12]$. On the contrary, the understanding of the modal volume has been more elusive, mostly due to the lack of theoretical and conceptual tools to normalize the optical modes of arbitrary open systems [7]. Important progress has been achieved on the description of photonics as a non-Hermitian problem via quasinormal modes (QNMs) with complex eigenvalues, which led to a deep modification of the common picture for $V$. The concept of a complex-valued $V$, with its imaginary part linked to the non-Hermitian nature of open systems [13] is now established and has recently been confirmed experimentally with near-field $Q$-perturbation experiments [14]. Non-Hermitian theories also predict that the complex nature of $V$ leads to a strongly non-Lorentzian spectra profile of the LDOS in peculiar photonic and plasmonic systems [13,15-18]. In coupled resonators, the spectral dependence of the Purcell enhancement is predicted to be a generalized Fano profile with a dip in the spectral line shape [13], similar to electromagnetic induced transparency and Autler-Townes splitting spectra [19-21]. However, an experimental demonstration of Fano-like LDOS is still lacking, together with a complete understanding of the many quantities playing a role in non-Hermitian photonics of coupled systems.

Here, we experimentally demonstrate LDOS with strong non-Lorentzian line shapes in pairs of coupled, high- $Q$ and small- $V$ photonic crystals cavities (PhCCs). We show that the LDOS spectral line shape and spatial distribution can be measured by near-field hyperspectral imaging. Pronounced non-Lorentzian line shapes are observed in agreement with numerical simulations, allowing us to reconstruct the near-field spatial distribution of the QNM modal volumes with comparable real and imaginary parts. We benchmark an analytical model to provide insight into the quantities playing a role in non-Lorentzian LDOS and QNM complex modal volumes. Finally, we demonstrate that non-Lorentzian line shapes can also be evidenced in far-field microphotoluminescence $(\mu \mathrm{PL})$ experiments with extended PhCCs, enabling a fine mode tuning and the measurement of the LDOS in the time domain.

The theoretical framework of the present study considers a quantum emitter with position $\mathbf{r}$ and dipole moment $\mathbf{p}=p \mathbf{u}$, with $\mathbf{u}$ the dipole direction, embedded in a photonic cavity. Within QNM theory, the cavity is described by a set of modes with normalized electric fields $\tilde{\mathbf{E}}_{n}(\mathbf{r})$ and 
complex frequencies $\tilde{\omega}_{n}=\omega_{n}-i \Gamma_{n}$, with $\omega_{n}$ the resonant frequency and $\Gamma_{n}$ the coherence damping rate [7]. In this framework, the spontaneous emission rate $\gamma(\mathbf{r}, \omega)$ (proportional to $\rho(\mathbf{r}, \omega)$ ) can be written as $\gamma(\mathbf{r}, \omega)=\sum_{n} \gamma_{n}(\mathbf{r}, \omega)$, where $\gamma_{n}$ is the contribution of the $n$th mode, and is given by [13]

$$
\begin{aligned}
\gamma_{n}(\mathbf{r}, \omega) & =-\frac{2 \omega}{\hbar}|\mathbf{p}|^{2} \operatorname{Im}\left[\frac{\left(\tilde{\mathbf{E}}_{n}(\mathbf{r}) \cdot \mathbf{u}\right)^{2}}{\left(\omega-\omega_{n}+i \Gamma_{n}\right)}\right] \\
& =\frac{2}{\hbar \varepsilon_{0} \varepsilon_{r}(\mathbf{r})}|\mathbf{p}|^{2} \operatorname{Re}\left[\frac{Q_{n}}{\tilde{V}_{n}(\mathbf{r})}\right] \frac{\omega}{\omega_{n}} \frac{\Gamma_{n}^{2}}{\left(\omega-\omega_{n}\right)^{2}+\Gamma_{n}^{2}}\left(1+\frac{\operatorname{Im}\left[\tilde{V}_{n}(\mathbf{r})\right]}{\operatorname{Re}\left[\tilde{V}_{n}(\mathbf{r})\right]} \frac{\omega-\omega_{n}}{\Gamma_{n}}\right) \\
& =\frac{2}{\hbar \varepsilon_{0} \varepsilon_{r}(\mathbf{r})}|\mathbf{p}|^{2} \frac{Q_{n}}{\left|\tilde{V}_{n}(\mathbf{r})\right|} \frac{\omega}{\omega_{n}}\left[\frac{\Gamma_{n}}{\left(q_{n}(\mathbf{r})^{2}+1\right)} \frac{\left(q_{n}(\mathbf{r})^{2}-1\right) \Gamma_{n}+2 q_{n}(\mathbf{r})\left(\omega-\omega_{n}\right)}{\left(\omega-\omega_{n}\right)^{2}+\Gamma_{n}^{2}}\right] .
\end{aligned}
$$

Note in Eq. (1a) that the electric field projected on the dipole moment direction is squared and not absolute squared, in contrast with classical textbook expressions [22]. The electric field of a leaky resonance is complex valued in general. To reach Eq. (1b), we introduced the complex-valued modal volume $\tilde{V}_{n}(\mathbf{r})=$ $\left[2 \varepsilon_{o} \varepsilon(\mathbf{r})\left(\tilde{\mathbf{E}}_{n}(\mathbf{r}) \cdot \mathbf{u}\right)^{2}\right]^{-1}$ and $Q_{n}=\frac{1}{2} \omega_{n} / \Gamma_{n} \quad$ [13]. The expression shows that all modal contributions to the emission rate exhibit a non-Lorentzian line shape, with deviations from the Lorentzian case on the order of $\operatorname{Im}\left[\tilde{V}_{n}(\mathbf{r})\right] / \operatorname{Re}\left[\tilde{V}_{n}(\mathbf{r})\right]$. Interestingly, this aspect of nonHermiticity is further stressed in Eq. (1c), where the term in squared parenthesis is a standard Fano profile [19-21]. The Fano parameter $q_{n}(\mathbf{r})=-\operatorname{Re}\left[\left(\tilde{\mathbf{E}}_{n}(\mathbf{r}) \cdot \mathbf{u}\right)\right] /$ $\operatorname{Im}\left[\left(\tilde{\mathbf{E}}_{n}(\mathbf{r}) \cdot \mathbf{u}\right)\right]$ is associated to the spatial phase of the $n$th QNM, which is a physical observable fully defined by the normalization. We stress the analogy with quantum mechanics, where Fano line shapes were discovered in the absorption of quasibound states in the continuum [23]. This is indeed the basis of non-Hermitian theory in photonics, where even the best photonic resonator, due to its losses in the far field, is a quasibound state in a continuum.

A counterintuitive effect of Eq. (1) is that, while the total emission rate $\gamma$ should always be positive at all frequencies, $\gamma_{n}$ can be negative in some frequency range. This possibility directly stems from the fact that QNMs are intrinsically linked. In a classical picture, the LDOS measures the radiation reaction acting on the emitter via the AbrahamLorentz friction force on the oscillating dipole charges [24]. Even if the total friction must always damp the motion $[\gamma(\mathbf{r}, \omega)>0], \gamma_{n}(\mathbf{r}, \omega)<0$ physically means that the backaction of the $n$th QNM exerts a negative friction (i.e., tends to increase the velocity) that reduces the total radiative damping. It is an interference effect occurring in the near field. In practice, the positivity of the LDOS suggests that a spectrally isolated mode should yield a $\gamma_{n}$ with a Lorentzian line shape (i.e., $\left[\tilde{\mathbf{E}}_{n}(\mathbf{r}) \cdot \mathbf{u}\right]$ is almost real), while two or more spectrally overlapping modes may yield $\gamma_{n}$ with strongly non-Lorentzian line shapes. This possibility was already predicted numerically in Ref. [13] with two monomode photonic-crystal cavities evanescently coupled with unbalanced $Q$ factors $\left(Q_{1}=90\right.$ and $\left.Q_{2}=300\right)$. Here, we experimentally demonstrate these predictions and show that their validity is not limited by any upper limit of the $Q$ factor.

We investigate two coupled $L 3$ cavities in a GaAs slab with unbalanced losses, and with high-density InAs quantum dots (QDs) embedded (details are given in the Supplemental Material [25]). From now on, labels "+" "and "-"refer to the QNMs of the coupled system, while labels "1" and "2" refer to the QNMs of the uncoupled cavities. The SEM image of one sample is reported in Fig. 1(a). The individual cavity losses and the frequency detuning are controlled, respectively, by changing the diameter of the air holes circled in purple and in orange $\left(200 \leq Q_{1} \leq 700\right.$, $Q_{2} \sim 6000$ ). The different samples are described by the parameters $z=\left|\left(\Gamma_{1}-\Gamma_{2}\right) / 2 g\right|$ and $\Delta=\left(\omega_{1}-\omega_{2}\right) /|2 g|$, with $g$ the coupling constant between the two uncoupled cavities and $\left(\omega_{1}, \omega_{2}\right)$ and $\left(\Gamma_{1}, \Gamma_{2}\right)$ their frequencies and loss rates, which are estimated by FDTD simulations (see Supplemental Material [25]). At $\Delta=0$, the condition $z<1(z>1)$ indicates strong (weak) coupling, while $z=1$ corresponds to an exceptional point where the two eigenvalues and eigenvectors coalesce $[19,20]$.

Measuring the absolute value of the LDOS requires lifetime and radiative efficiency measurements [26]. Absolute measurements are, however, not necessary for our scope. We propose here that near-field photoluminescence (SNOM-PL) experiments enable measuring relative changes of the spectral line shape and spatial distribution of the LDOS at the tip position. Our claim is supported by FDTD simulations performed on a wide range of $\mathrm{PhCC}$ systems (see Supplemental Material [25]). Examples of SNOM-PL spectra measured at the center of the PhCCs are given in Figs. 1(b)-1(e) for different nominal $z$ (and nominal $\Delta \simeq 0$ ), ranging from strong to weak coupling. Hereafter, the photon energy $(E=\hbar \omega)$ is used instead of 

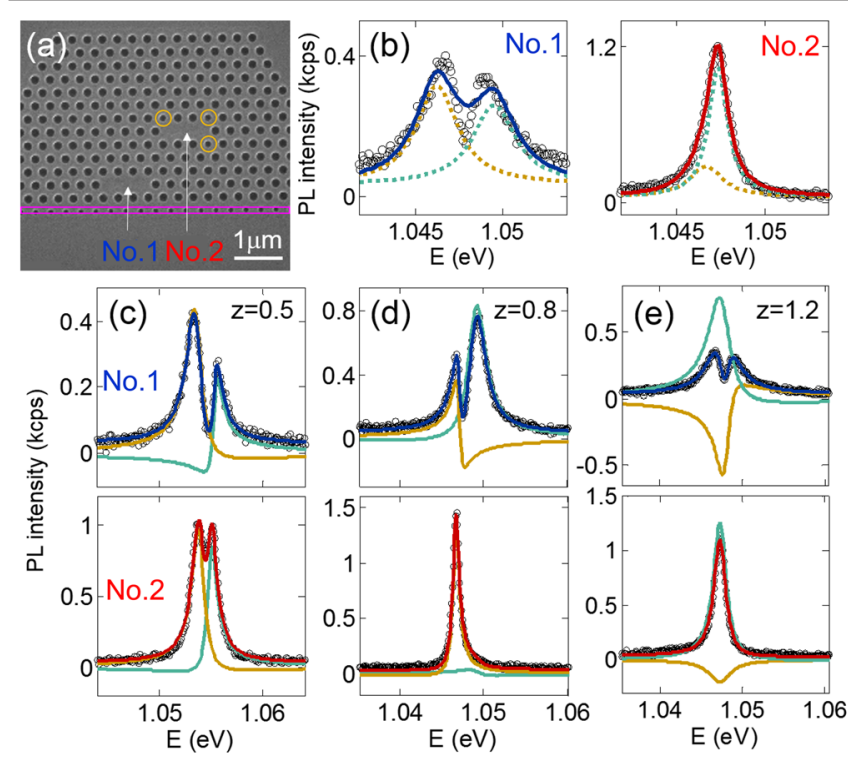

FIG. 1. (a) SEM image of one sample with nominal $z=0.8$; the modified air holes are highlighted with different colors. Cavities No. 1 and No. 2 are labeled with color code (blue and red) which is used also in the fits. (b) Experimental SNOM-PL spectra (open circles) in the center of cavities No. 1 and No. 2 (left and right panel) with best Lorentzian fits with different $\tilde{\omega}_{ \pm}$(red and blue lines) and the two Lorentzian components (dark yellow and green dotted lines). (c)-(e) Experimental SNOM-PL spectra (open circles) for systems with different nominal $z$ and $\Delta \simeq 0$. Upper (lower) panels are data taken in the center of cavities No. 1 and No. 2 together with the fits from Eq. (1) (blue and red lines). In all panels, we report the resulting "+" and "-"QNM contributions (dark yellow and green full lines).

the frequency $\omega$. The LDOS spectra in cavity No. 1 exhibit a double-peaked line shape and the spectra in cavity No. 2 are double peaked for $z \lesssim 0.5$ and single peaked for $z \gtrsim 0.5$. To evidence the non-Lorentzian LDOS line shape, we report the best fits of the two LDOS spectra in Fig. 1(b) with two Lorentzians (solid lines, while dotted lines are the mode decomposition). Even allowing the fitted $\tilde{\omega}_{ \pm}$to differ in the two cavities, which is unphysical, the spectrum in cavity No. 1 cannot be accurately fitted by the sum of two Lorentzians. The best fits with identical $\tilde{\omega}_{ \pm}$(not shown) are worse; therefore even small deviations from Lorentzian $\rho(\mathbf{r}, \omega)$ emerge when simultaneously fitting data from both cavities.

The PL intensity in the near field for three different samples are reported in Figs. 1(c)-1(e); the upper and lower panel refer to PL detected in cavities No. 1 and No. 2, respectively. As predicted by the QNM model, we observe that the LDOS in cavity No. 1 has an evident nonLorentzian shape. Data are well described by the QNM formalism as the sum of two Fano profiles [blue and red lines are fits from Eq. (1) and the dark yellow and green lines are the mode decomposition in Fano profiles]; note that the data in Fig. 1(e) coincide with the one in Fig. 1(b). It is worth stressing that Lorentzian fits with different $\tilde{\omega}_{ \pm}$

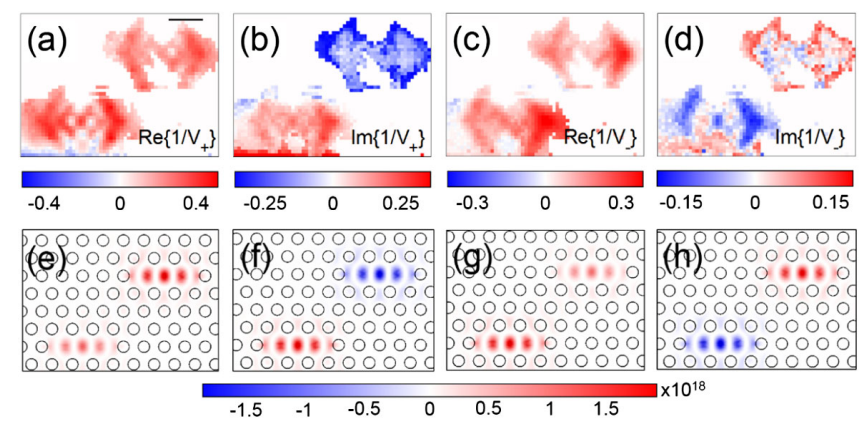

FIG. 2. (a)-(d) SNOM-PL maps (in a sample with $z=0.5$ ) of the real and imaginary parts of the parameters $1 / V_{ \pm}(\mathbf{r})$ obtained by fitting of the SNOM-PL spectra in different points. The color scales are in arbitrary units but identical in all figures. (e)-(h) QNM predictions for the same nominal PhCC parameters. The color scale is in MKSA units.

[Fig. 1(b)] and Fano fits [Figs. 1(c)-1(e)] use the same number of free parameters (see Supplemental Material [25], Sec. S2). The fits in Figs. 1(c)-1(e) reproduce well the data and unveil the possibility for resonances to have negative contributions to the LDOS. To our knowledge, this constitutes the first experimental demonstration of strong nonLorentzian LDOS of individual photonic resonances, which is a hallmark of non-Hermiticity [13].

Interestingly, our method allows us to globally map the spatial distribution of $\rho(\mathbf{r}, \omega)$ inside and outside the cavities with a spatial resolution of the order of $130 \mathrm{~nm}=\lambda / 10$. For this aim, we fit the SNOM-PL measurements with Eq. (1) at every probed point. The fits are performed allowing tiny variations around the determined parameters to include the tip-induced frequency shift and loss-rate variations of the resonances [22]. The spatial maps of the real and imaginary parts of $1 / V_{ \pm}(\mathbf{r})$ are compared with the predictions from QNM theory in Figs. 2 (for a sample with $z=0.5$ ). The QNMs were computed and normalized using the QNM solver QNMEig [27] of the freely available package MAN (Modal Analysis of Nanoresonators) [28]. The experimental measurements agree with the theoretical predictions in terms of the mode symmetry and the ratio between the imaginary and real parts of the inverse mode volumes $(\approx 1 / 2)$. We conclude that SNOM-PL measurements provide subwavelength maps of the complex modal volumes of overlapping QNMs from the phase information encoded in the Fano line shape [i.e., in $q_{+}(\mathbf{r})$ or in the complex volume $V_{ \pm}(\mathbf{r})$, see Supplemental Material [25], Sec. S2].

Experimental measurements (and FDTD simulations, see Ref. [25]) demonstrate that the LDOS of coupled systems shows a large variety of Fano line shapes. QNM theory via Eq. (1) provides a rigorous framework to analyze the modes of complex systems. However, we aim to get an intuitive picture that clarifies the role of the different physical parameters in coupled systems. Therefore, we developed a simplified analytical model based on the well-known coupled-mode model [19-21,23,26,29] 


$$
\left(\begin{array}{cc}
-\omega+\tilde{\omega}_{1} & g \\
g & -\omega+\tilde{\omega}_{2}
\end{array}\right)\left(\begin{array}{l}
c_{1} \\
c_{2}
\end{array}\right)=\left(\begin{array}{l}
P_{1} \\
P_{2}
\end{array}\right)
$$

Here, $c_{j}$ represents the electric field amplitude in the $j$ th cavity and $g$ the field tunneling rate among the two cavities (here assumed to be real) [29]. The driving term $P_{j}$ represents the field injection per unit of time in the $j$ th cavity mode, which describes either the excitation via optical pumping or the emission of a dipole. Under the additional assumption that $g$ is independent of the frequency, the homogeneous solution $\left(P_{1}=P_{2}=0\right)$ leads to two QNM eigenvalues $\tilde{\omega}_{ \pm}=\omega_{ \pm}-i\left(\Gamma_{ \pm} / 2\right)=\frac{1}{2}\left(\tilde{\omega}_{1}+\tilde{\omega}_{2}\right) \pm \frac{1}{2} \tilde{\Omega}$ for the coupled system with $\tilde{\Omega}=\sqrt{\left(\tilde{\omega}_{1}-\tilde{\omega}_{2}\right)^{2}+4 g^{2}}$ the complex frequency splitting.

When the $j$ th cavity mode is driven $\left(P_{1} \neq 0, P_{2}=0\right.$ or $P_{1}=0, P_{2} \neq 0$ ), e.g., by placing the emitter at an antinode of the $j$ th cavity mode, the spectral dependence of $\operatorname{Im}\left(c_{j} / P_{j}\right)$ is representative of the LDOS line shapes inside the $j$ th cavity [(hereafter $\rho_{j}(\omega) \equiv \rho\left(\mathbf{r}=\mathbf{r}_{j}, \omega\right)$ ] [29] (see the FDTD simulations in the Supplemental Material [25]):

$\rho_{j}(\omega) \propto-\operatorname{Im}\left[\frac{c_{j}(\omega)}{P_{j}}\right]=-\operatorname{Im}\left[\frac{1 / V_{j,+}}{\left(\omega-\tilde{\omega}_{+}\right)}+\frac{1 / V_{j,-}}{\left(\omega-\tilde{\omega}_{-}\right)}\right]$,

where we defined

$\frac{1}{V_{1, \pm}}=\frac{2 \tilde{\Omega} \pm\left(\tilde{\omega}_{1}-\tilde{\omega}_{2}\right)}{4 \tilde{\Omega}} ; \quad \frac{1}{V_{2, \pm}}=\frac{2 \tilde{\Omega} \mp\left(\tilde{\omega}_{1}-\tilde{\omega}_{2}\right)}{4 \tilde{\Omega}}$.

By comparing with Eq. (1), each coupled QNM contributes to the modal LDOS $\rho_{j}(\omega)$ with distinct Fano terms $\rho_{j, \pm}(\omega) \propto-\operatorname{Im}\left\{\left[1 / V_{j, \pm}\right] /[(\omega-\tilde{\omega} \pm)]\right\}$, and then $V_{j, \pm}(j=$ $1,2)$ are proportional to the mode volumes on the QNMs in the $j$ th cavity. Although this phenomenological model misses several features of the rigorous QNM theory [13], e.g., the spatial dependence of the modal volume, it provides analytical expressions of $V_{j, \pm}$ as a function of the parameters $\tilde{\omega}_{j}(j=1,2)$ and $g$, thereby enabling a comprehensive analysis of non-Lorentzian LDOS line shapes.

We first validate the phenomenological model by comparing the LDOS modal decomposition obtained with rigorous QNM calculations. The top panel in Fig. 3(a) shows the normalized QNMs computed with QNMEig to further inject the resulting values of $V_{ \pm}$into Eq. (1). The sum (blue line) of the two mode contributions (dark yellow and green dotted lines) reproduces the total LDOS computed with FDTD (open circles) without any fitting parameter. This expected agreement [13] is used to validate the analytical model predictions recovered from Eqs. (3)-(4) and shown in the bottom panel. Conclusively, the fitted line shapes of the individual mode contributions match those directly found with the QNM theory. The

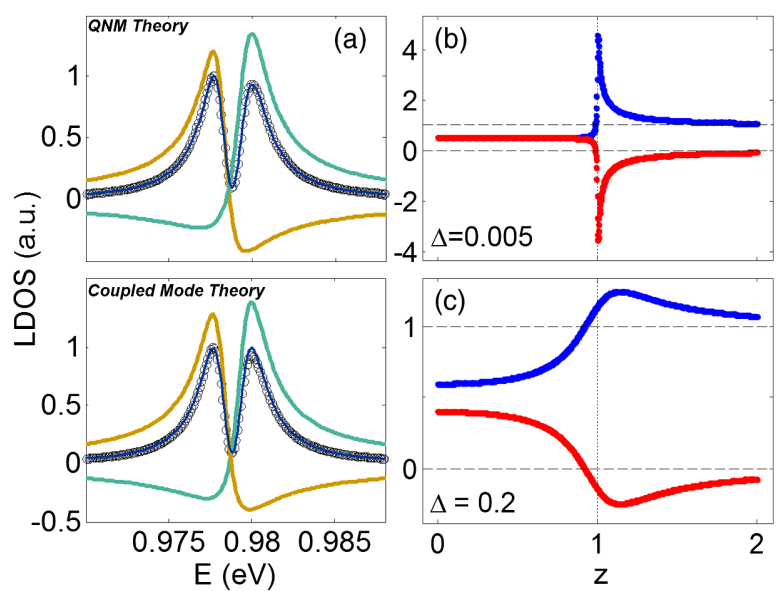

FIG. 3. (a) Top panel: FDTD-LDOS simulation (open circles) compared with rigorous QNM theory (blue line) and its mode decomposition (dark yellow and green dotted lines). Bottom panel: FDTD-LDOS simulation (open circles) compared with Eq. (3) (bottom panel) (blue line) and its mode decomposition (dark yellow and green dotted lines). (b) Real part of $\operatorname{Re}\left\{1 / V_{1,+}\right\}$ (blue dots) and $\operatorname{Re}\left\{1 / V_{1,-}\right\}$ (red dots), computed by Eq. (4) as a function of $z$ with almost no detuning. (c) Real part of $\operatorname{Re}\left\{1 / V_{1,+}\right\}$ (blue dots) and $\operatorname{Re}\left\{1 / V_{1,-}\right\}$ (red dots), computed by Eq. (4) as a function of $z$ with $\Delta=0.2$.

proportionality factor is the only free parameter when fitting Eq. (3) with the FDTD data, since $\tilde{\omega}_{ \pm}$and $V_{j, \pm}$ are determined from $\tilde{\omega}_{j}$, and $g$ given by the calibration simulations (details in the Supplemental Material [25]). However, in Fig. 3(a) we improved the fit quality by allowing tiny additional variations of $\tilde{\omega}_{j}$ and $g$ in the coupled system with respect to the calibration parameters $\left(<1 \%\right.$ for $\tilde{\omega}_{j}$ and $<10 \%$ for $\left.g\right)$, accounting for the impossibility to perfectly identify the parameters of the uncoupled cavities. After this numerical benchmark (see Ref. [25]), we explored how the input parameters impact the modal volumes and LDOS line shapes. The coupledmode parameters of the analytical model $\tilde{\omega}_{j}$ and $g$ are calibrated via FDTD simulations with varying structural parameters. The analytical model predicts that by fixing three parameters $\left(z, \Delta\right.$, and $\left.y=|2 g| / \Gamma_{2}\right)$, the LDOS line shape is invariant in the normalized frequency scale $\left(\omega-\omega_{o}\right) / \Gamma_{2}$. Therefore, strong non-Lorentzian LDOS can be expected even in cavities with extremely high $Q$ if the detuning in frequency and losses are controlled to keep $z, \Delta$, and $y$ constant.

Important information on the QNM modal volumes can be obtained from Eq. (4), helping to interpret the experimental data in Fig. 2. We highlight three relevant observations for this case $(z=0.5$ and $\Delta=0.2)$ : (i) in each cavity, $\operatorname{Im}\left\{1 / V_{+}(\mathbf{r})\right\}$ and $\operatorname{Im}\left\{1 / V_{-}(\mathbf{r})\right\}$ have opposite signs; (ii) for each QNM, $\operatorname{Im}\left[1 / V_{ \pm}(\mathbf{r})\right]$ changes sign moving from one cavity to the other; and (iii) $\operatorname{Re}\left\{1 / V_{+}(\mathbf{r})\right\}$ and $\operatorname{Re}\left\{1 / V_{-}(\mathbf{r})\right\}$ are positive in almost all points. These features are predicted by rearranging Eq. (4) so 


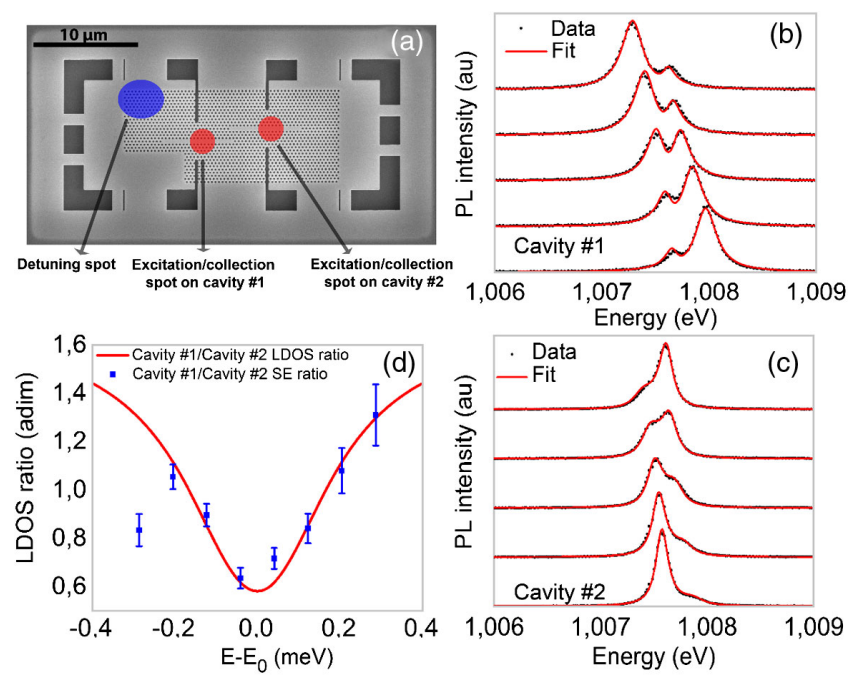

FIG. 4. (a) SEM picture of the system. The circles indicate the position of the detuning (blue) and excitation or collection lasers (red). Air trenches around the structure suppress lateral heat diffusion and maximize thermal shift. (b) Collection of far-field photoluminescence spectra from cavity No. 1 at different detuning conditions (black points), and corresponding fit (red curves). (c) Same as in (b), but from cavity No. 2. (c) $\rho_{1}(\omega) / \rho_{2}(\omega)$ (red line), and ratio of the measured SE rates (blue points) at $\Delta=0$. The data are plotted vs the energy detuning, where the zero corresponds to the photon energy $E_{o}$ at the PL dip.

that $\operatorname{Im}\left\{1 / V_{1, \pm}\right\}=-\operatorname{Im}\left\{1 / V_{2, \pm}\right\} \quad$ and $\operatorname{Im}\left\{1 / V_{j,+}\right\}=$ $-\operatorname{Im}\left\{1 / V_{j,-}\right\}$, in agreement with points (i) and (ii) for any parameter values. Therefore, two QNM contributions to the total $\rho(\mathbf{r}, \omega)=\rho_{+}(\mathbf{r}, \omega)+\rho_{-}(\mathbf{r}, \omega)$ tend to have specular line shapes, as shown in Fig. 3(a). The physical reason is that $\rho_{+}(\mathbf{r}, \omega)<0$ implies $\rho_{-}(\mathbf{r}, \omega)>0$, and vice versa. For point (iii), we numerically show that $\operatorname{Re}\left\{1 / V_{j, \pm}\right\}>0(j=1,2)$ holds in the strong coupling regime $(z<1)$ and small $\Delta$. Results for $\operatorname{Re}\left\{1 / V_{1, \pm}\right\}$ for different $z$ and for $\Delta=0.005$ and $\Delta=0.2$ are reported in Figs. 3(b)-3(c), respectively. Please note that the analytical model only gives values proportional to $1 / V_{n, \pm}$ whereas rigorous QNM computations are needed for quantitative predictions; other details on the comparison between QNM computations and the coupled mode model are given in the Supplemental Material [25] (Secs. S2 and S5).

To further confirm that the individual modal contributions to the LDOS in coupled cavities is non-Lorentzian even for ultrahigh $Q$ (nominal $Q=40000$ ), we studied coupled L20 PhCCs (20 holes missing in a row), which enable excitation of a spatial region with almost uniform LDOS by $\mu$ PL. Within this condition, we demonstrate that even the far-field QD spectrum reflects the non-Lorentzian LDOS (see Supplemental Material [25]). Figure 4(a) reports the SEM of the coupled system (designed to have $\approx 50 \%$ loss mismatch) with the spots for detection and for localized thermal tuning by optical pumping, which allows us to continuously change the detuning $\Delta[30]$.
The $\Delta$-dependent spectra at a temperature of $T=77 \mathrm{~K}$ [Figs. 4(b)-4(c)] well agree with the curves obtained by using Eq. (3) (see Supplemental Material [25]), while a fit with a sum of Lorentzians fails when the same values of $\tilde{\omega}_{ \pm}$ are considered in both cavities. Furthermore, time-resolved experiments are performed to directly measure the changes in the $\gamma_{j}(\omega)$ (see Supplemental Material [25]). Figure 4(d) shows the energy-dependent ratios of the experimental SE rates at $\Delta=0$ in (cavity No. 1 and cavity No. 2, blue points) vs the quantity $\rho_{1}(\omega) / \rho_{2}(\omega)$ (red curve) from the PL spectra fits, showing very good agreement. We stress that a Lorentzian model of the LDOS is expected to predict the same spectral line shapes in the two cavities at $\Delta=0$, and a constant $\gamma_{1}(\omega) / \gamma_{2}(\omega)$. These features prove that deviations from Lorentzian behavior can be experimentally addressed with the far-field detection of PL and high- $Q$ cavities.

In conclusion, we provided extensive experimental evidence, including near-field, far-field, and time-resolved data, of the existence of non-Lorentzian LDOS in systems consisting of two PhCCs with relatively low optical radiation loss. In agreement with QNM theory, we found that the LDOS of each mode deviates from Hermitian theory and exhibits a strongly non-Lorentzian profile, including negative values. Our SNOM approach also provides a direct measure of the near-field maps of the complex mode volumes of both QNMs with subwavelength spatial resolutions. These results mark a significant step forward in the understanding of the non-Hermitian nature of open systems, which are not limited to photonics or plasmonics, but also can be relevant in many fields of quantum mechanics [31], including nanoelectronics.

D. P. and A. F. acknowledge funding from the Netherlands Organisation for Scientific Research (NWO), and thank Armando Genco and Michele Cotrufo for the helpful discussions. D. B. and F. I. acknowledge Niccolò Mignani for his patient and fruitful collaboration. P. L. thanks J.-P. Hugonin and C. Sauvan for interesting discussions on the Purcell effect and the concept of complex volumes. M. G. acknowledges financial support from NWO for visits at TU/e and funding from CdR Firenze (2018/24257). F. I. acknowledges funding from NARCISO (EU-H2020, ID:828890). P. L. acknowledges NOMOS (ANR-18CE240026) and I-SQUAD (ANR-18CE47-0006) projects for financial support.

D. P. and D. B. contributed equally to this work.

Note added in proof.-Recently, we became aware of an independent experimental demonstration of non-Lorentzian line shapes in hybrid antenna-cavity systems [32].

[1] R. Soref, APL Photonics 3, 021101 (2018).

[2] J. R. Mejía-Salazar, and O. N. Oliveira, Chem. Rev. 118, 10617 (2018). 
[3] S. Pirandola, B. R. Bardhan, T. Gehring, C. Weedbrook, and S. Lloyd, Nat. Photonics 12, 724 (2018).

[4] M. Kadic, G. W. Milton, M. van Hecke, and M. Wegener, Nat. Rev. Phys. 1, 198 (2019).

[5] A. J. Shields et al., Nat. Photonics 1, 215 (2007).

[6] T. Ozawa, Rev. Mod. Phys. 91, 015006 (2019).

[7] P. Lalanne, W. Yan, K. Vynck, C. Sauvan, and J.-P. Hugonin, Laser Photonics Rev. 12, 1700113 (2018).

[8] M. Pelton, Nat. Photonics. 9, 427 (2015).

[9] R. Carminati et al., Surf. Sci. Rep. 70, 1 (2015).

[10] E. M. Purcell, H. C. Torrey, and R. V. Pound, Phys. Rev. 69, 37 (1946).

[11] Y. Akahane, T. Asano, B.-S. Song, and S. Noda, Nature (London) 425, 944 (2003).

[12] Y. Lai et al., Appl. Phys. Lett. 104, 024110 (2014).

[13] C. Sauvan, J. P. Hugonin, I. S. Maksymov, and P. Lalanne, Phys. Rev. Lett. 110, 237401 (2013).

[14] K.-G. Cognée, W. Yan, F. La China, D. Balestri, F. Intonti, M. Gurioli, A. F. Koenderink, and P. Lalanne, Optica 6, 269 (2019).

[15] Q. Bai, M. Perrin, C. Sauvan, J.-P. Hugonin, and P. Lalanne, Opt. Express 21, 27371 (2013).

[16] E. A. Muljarov and W. Langbein, Phys. Rev. B 94, 235438 (2016).

[17] G. Kewes, F. Binkowski, S. Burger, L. Zschiedrich, and O. Benson, ACS Photonics 5, 4089 (2018).

[18] R. Faggiani, J. Yang, R. Hostein, and P. Lalanne, Optica 4, 393 (2017).

[19] N. Caselli et al., Nat. Commun. 9, 396 (2018).

[20] B. Peng, Ş. K. Özdemir, W. Chen, F. Nori, and L. Yang, Nat. Commun. 5, 5082 (2014).
[21] M. F. Limonov, M. V. Rybin, A. N. Poddubny, and Y. S. Kivshar, Nat. Photonics 11, 543 (2017).

[22] S. V. Gaponenko, Introduction to Nanophotonics (Cambridge University Press, Cambridge, 2017).

[23] U. Fano, Phys. Rev. 124, 1866 (1961).

[24] see, for instance, D. J. Griffiths, Introduction to Electrodynamics (Cambridge University Press, Cambridge, 2017), Chap. 11.

[25] See Supplemental Material at http://link.aps.org/ supplemental/10.1103/PhysRevLett.124.123902 for details on the design of the devices, explicit calculations, FDTD simulations, QNM computations, far-field data and fit.

[26] M. Munsch, A. Mosset, A. Auffèves, S. Seidelin, J. P. Poizat, J.-M. Gérard, A. Lemaître, I. Sagnes, and P. Senellart, Phys. Rev. B 80, 115312 (2009).

[27] W. Yan, R. Faggiani, and P. Lalanne, Phys. Rev. B 97, 205422 (2018).

[28] QNM solvers and toolboxes are freely available online at https:// www.lp2n.institutoptique.fr/light-complex-nanostructures.

[29] A. Pick, B. Zhen, O. D. Miller, C. W. Hsu, F. Hernandez, A. W. Rodriguez, M. Soljačić, and S. G. Johnson, Opt. Express 25, 12325 (2017).

[30] H. M. H. Chong and R. M. De La Rue, IEEE Photonics Technol. Lett. 16, 1528 (2004).

[31] See, for instance, N. Moiseyev, Non-Hermitian Quantum Mechanics (Cambridge University Press, Cambridge, 2011).

[32] H. M. Doeleman, Ph.D. thesis, University of Amsterdam, 2019, Chap. 7. 